\title{
Beyond KRAS: Practical Molecular Targets in Pancreatic Adenocarcinoma
}

\author{
Albert Grinshpun $^{\mathrm{a}} \quad$ Yonaton Zarbiv $^{\mathrm{a}} \quad$ Jason Roszik $^{\mathrm{b}} \quad$ Vivek Subbiah $^{\mathrm{c}}$ \\ Ayala Hubert ${ }^{a}$ \\ aSharett Institute of Oncology, Hadassah-Hebrew University Medical Center, \\ Jerusalem, Israel; b Melanoma Medical Oncology and Genomic Medicine, The University of \\ Texas MD Anderson Cancer Center, Houston, TX, USA; 'Department of Investigational \\ Cancer Therapeutics, Division of Cancer Medicine, The University of Texas MD Anderson \\ Cancer Center, Houston, TX, USA
}

\section{Keywords}

Pancreatic adenocarcinoma $\cdot \mathrm{KRAS} \cdot \mathrm{BRAF} \cdot \mathrm{RET} \cdot$ Targeted therapy

\begin{abstract}
Pancreatic adenocarcinoma (PDAC) has a grim prognosis. Molecular and genomic analyses revealed that the striking majority of these tumors are driven by KRAS mutation, currently not amenable to targeted therapy. However, other driver mutations were found in a small fraction of patients. Herein we report of 3 cases of patients with metastatic PDAC and wildtype KRAS, found to harbor BRAF or RET pathogenic alterations. The patients were treated with targeted therapies with variable success. In our opinion, those proof-of-concept cases argue in favor of additional research and clinical trials' effort in this small but significant PDAC population with uncommon driver mutations.

(C) 2019 The Author(s)

Published by S. Karger AG, Basel
\end{abstract}




\section{Case Reports in Oncology}

Case Rep Oncol 2019;12:7-13

DOI: $10.1159 / 000496018$ (c) 2019 The Author(s). Published by S. Karger AG, Basel www.karger.com/cro

Grinshpun et al:: Beyond KRAS: Practical Molecular Targets in Pancreatic Adenocarcinoma

\section{Introduction}

Pancreatic adenocarcinoma (PDAC) is a major cause of cancer-related mortality in Western countries. It is expected to become the second most lethal cancer within a decade [1]. The most common genomic alterations in PDAC are in KRAS oncogene (more than $93 \%$ of tumors), CDKN2A, SMAD4 and TP53 [2]. Importantly, all these mutations are not amenable to targeted therapies. The cornerstone of current first-line treatment of PDAC is a combination chemotherapy (such as protein-bound paclitaxel with gemcitabine or oxaliplatin-based protocols). Despite extensive research, there are only two FDA-approved targeted agents for PDAC: Erlotinib (EGFR inhibitor with very modest impact on survival) and the check-point immunotherapeutic agent Pembrolizumab (anti-PD1) in case of mismatch-repair (MMR) deficient tumors [3].

BRAF is another well-described oncogene, mutated in $15 \%$ of all tumors, mainly in melanoma, papillary thyroid carcinoma and hairy-cell leukemia [4]. Up to $90 \%$ of BRAF mutations are comprised of the single substitution of glutamic acid to valine at codon 600 (V600E). This change cause constitutive activation of the kinase domain and further oncogenic effects. Recently published comprehensive molecular analysis of PDAC by The Cancer Genome Atlas Research Network [2] show that about 3\% of tumors harbor BRAF alteration (and KRAS wildtype). Fortunately, potent inhibitors of BRAF V600E were developed (Dabrafenib, Vemurafenib, etc.) and have shown significant clinical utility. There are only two published reports of BRAF-mutated PDAC patients $(n=5)$ treated with Vemurafenib, resulting in four minimal tumor responses and one partial response (in a patient with CUX1-BRAF fusion) [5, 6].

Oncogenic RET aberrations are either germline or somatic mutations or translocations [7]. Heritable mutations cause multiple endocrine neoplasia type 2 (MEN2) and somatic mutations are well described in papillary thyroid cancer, lung adenocarcinoma and chronic myelomonocytic leukemia. RET is expressed in up to $65 \%$ of PDAC and probably involved in the metastatic process. RET inhibitors are being tested in clinical trials, however recent data does not support RET inhibition without a biomarker [8].

Here we present a case of RET-mutated and two cases of BRAF-mutated PDAC (all KRAS wildtype) patients treated with a specific targeted therapies.

\section{Case Reports}

First Case

75-year-old female patient, with no significant comorbidities or family history of cancer, presented to the Emergency Department with severe abdominal pain and progressive weight loss. Computed Tomography (CT) revealed multiple hepatic lesions, omental nodules and pancreatic mass. CA19-9 was $918 \mathrm{u} / \mathrm{mL}$. Liver biopsy revealed poorly differentiated pancreatic carcinoma, mismatch repair (MMR) proficient, without BRCA1/2 mutations. Surprisingly, tumor mutation testing using next-generation-sequencing (NGS) panel discovered wildtype KRAS and pathogenic BRAF c.1799T $>$ A (V600E) mutation. The patient received a single cycle of Oxaliplatin-based chemotherapy and decided not to pursue additional cycles due to poor tolerability. The case was presented at a multidisciplinary tumor board (MTB) and combination targeted therapy using Dabrafenib and Trametinib was suggested. The patient was given 


\section{Case Reports in Oncology}

Case Rep Oncol 2019;12:7-13

DOI: $10.1159 / 000496018$

(C) 2019 The Author(s). Published by S. Karger AG, Basel www.karger.com/cro

Grinshpun et al:: Beyond KRAS: Practical Molecular Targets in Pancreatic Adenocarcinoma

full dose therapy with Dabrafenib $150 \mathrm{mg}$ twice daily with Trametinib $2 \mathrm{mg}$ once a day. During treatment the patient had no apparent side effects. CA19-9 declined within a week from 2,774 to $1,687 \mathrm{u} / \mathrm{mL}$. After 19 days on treatment schedule, the patient has arrived to the Emergency Department with severe abdominal pain. CT showed intra-peritoneal free air without obvious anatomic pathology involving the intestines. Disease extent evaluation was difficult due to lack of oral and intravenous contrast media. Sadly, the patient refused to undergo exploratory surgery and deceased within several hours.

\section{Second Case}

56-year-old male was admitted for evaluation of prolonged abdominal pain and severe weight loss. He has congestive heart failure and no family history of cancer. CT scan revealed pancreatic mass, omental implants and multiple hepatic lesions. CA19-9 was within normal limits $(18.5 \mathrm{u} / \mathrm{mL})$. Liver biopsy exhibited poorly differentiated carcinoma, suitable for pancreatic origin. Again, the tumor was MMR-proficient, wild-type sequences of BRCA1/2. Due to low performance status and intermediate cardiac function, the patient started monotherapy with gemcitabine. Meanwhile, NGS panel discovered wildtype KRAS and BRAF c.1799_1801delTGA mutation, likely pathogenic according to ClinVar database [9]. The case was discussed on MTB and the patient was advised to receive BRAF and MEK inhibitors on first sign of disease progression. Close cardiac monitoring was offered. As expected, metastatic progression was recorded and the patient started full dose Dabrafenib and Trametinib. After two weeks of treatment the patient developed severe unilateral interstitial lung disease. He received high dose steroids and all drugs except Dabrafenib were discontinued. The patient has recovered and resumed low dose Trametinib. The tumor was stable (per CT and CA19-9) for 3 months. However, after 3 additional months, just before his next evaluation the patient arrived to the Emergency Department in severe shock (probably septic) and passed away within minutes (post mortem analysis was not done).

\section{Third Case}

64-year-old male whose medical history was significant for type 2 diabetes, presented to the emergency department in our institution complaining of jaundice and weight loss. Lab results showed evidence of obstructive jaundice. Imaging showed pancreatic mass with no evidence of distant metastasis. A subsequent biopsy results were highly suggestive of ductal adenocarcinoma of the pancreas. CA19-9 at diagnosis was $400 \mathrm{u} / \mathrm{mL}$.

The patient received four rounds of neoadjuvant FOLFIRINOX chemotherapy and underwent pancriaticoduedonectomy. Pathology following surgery showed moderately to poorly differentiated adenocarcinoma of pancreatic head with vascular invasion. NGS (commercial FoundationOne test) detected RET rearrangement at exon 11 . The patient decided to forgo chemotherapy and received Cabozantinib. Prior to treatment, a PET scan showed no evidence of active disease. The patient was on Cabozantinib $100 \mathrm{mg}$ daily for 5 months. The treatment was well tolerated with mild acneiform rash. However, CA19-9 levels slowly increased while receiving this treatment, at last measurement it was 2,400 u/mL. Latest imaging revealed disease progression and therefore the patient re-started chemotherapy. 


\section{Case Reports in Oncology}

Case Rep Oncol 2019;12:7-13

DOI: $10.1159 / 000496018$ (c) 2019 The Author(s). Published by S. Karger AG, Basel www.karger.com/cro

Grinshpun et al.: Beyond KRAS: Practical Molecular Targets in Pancreatic Adenocarcinoma

\section{Discussion}

Currently, except MMR-deficiency, there are no clinically significant molecular targets in PDAC. To the best of our knowledge, here we present the first case reports of combination targeting of BRAF and its downstream effector MEK in a patient with metastatic PDAC.

BRAF V600E mutation is well-described biomarker for efficacy of BRAF inhibitors in melanoma. Multiple trials on various BRAF-mutant tumor types have shown that histology-agnostic approach is not always appropriate [4]. For example, the significant clinical benefit of BRAF inhibition in melanoma patients cannot be recapitulated in BRAF-mutated colorectal cancers. A landmark paper [6] showed that targeting the same so-called 'predictive' BRAF mutation in different non-melanoma tumors results in a wide range of response extents. Further research is required to elucidate these tissue specific factors that interact and modulate the response to BRAF inhibition. Another strategy to overcome resistance is adding MEK1/2 inhibitors to BRAF inhibitors. MEK is a downstream kinase in the MAPK pathway. Its inhibition has gained additional clinical benefit in colorectal and lung cancer (in terms of response rate and progression free-survival) and more meaningful prolongation of overall survival in metastatic melanoma [10-12], without significant addition of adverse effects.

RET oncogene is not typically mutated in PDAC. There are at least six multi-tyrosine kinase inhibitors that can inhibit RET: Lenvatinib, Cabozantinib, Vandetanib, Ponatinib, Sorafenib, Sunitinib and more experimental compounds [13]. Nowadays, there are no clinicallyproven methods to choose the best agent besides its availability, potential side effects and price. Nevertheless, preliminary results imply ex vivo systems as potential tools for this task [14].

BRAF and RET aberrations are detected in published projects of PDAC genome sequencing. For example, TCGA data [2] has 1.6\% (12/740 samples) and 1.5\% (11/740) alterations of BRAF and RET, respectively. Table 1 summarizes current data in the largest public databases: TCGA and GENIE $[2,15]$. Unfortunately, treatment and outcome data are not available.

As previously mentioned, in spite of dozens of randomized clinical trials, chemotherapeutic approach for treatment of PDAC achieved modest success. Furthermore, targeted agents trial were also disappointing [3]. There is a clear need for novel approaches, such as targeting the tumor microbiome, which was shown to inactivate chemotherapy [16]. Furthermore, $\mathrm{T}$ cell-based immunotherapies targeting neoantigens are potentially feasible in PDAC, however, only if the patient has the right human leukocyte antigen (HLA) type [17]. Another attractive option is biomarker-based targeted therapy. BRAF mutations in PDAC are relatively rare but potentially significant due to the availability of effective BRAF/MEK inhibitors. As a proof of principal in PDAC; Hainswoth et al. [5] show that targeting HER2, novel target in PDAC, with dual blockade can give promising response rate (two partial responses out of nine patients with HER2 amplification or mutation). We stress that performing trials of targeted therapies in PDAC with currently available agents may have clinical benefit in this poor-outcome population. 


\section{Case Reports in Oncology}

Case Rep Oncol 2019;12:7-13
\begin{tabular}{l|l}
\hline DOI: 10.1159/000496018 & $\begin{array}{l}\text { (c) 2019 The Author(s). Published by S. Karger AG, Basel } \\
\text { www.karger.com/cro }\end{array}$
\end{tabular}

Grinshpun et al.: Beyond KRAS: Practical Molecular Targets in Pancreatic Adenocarcinoma

\section{Conclusion}

In our opinion, the present report highlights two important issues in PDAC management: (1) the utility of NGS early in disease course in order to identify potentially targetable alterations in KRAS wildtype subset of patients (2) the deep need for additional clinical research of combination targeted therapies. It seems the incidence of BRAF mutation in PDAC resembles the ROS1 translocation in non-small cell lung cancer (NSCLC) [18]. Nowadays, ROS1 analysis is the standard of care in newly diagnosed NSCLC patients and embedded in national and global practice guidelines. Similarly, further research may add to consideration BRAF $\backslash$ RET analysis in KRAS wildtype PDAC patients (e.g. during this manuscript preparation two additional cases were identified).

In conclusion, identifying the precise molecular alteration and administration of active combination targeted therapy might have a potential activity in a small but significant portion of patients.

\section{Acknowledgments}

The drugs (Dabrafenib \& Trametinib) were kindly provided by the Novartis Company.

The authors would like to acknowledge the American Association for Cancer Research and its financial and material support in the development of the AACR Project GENIE registry, as well as members of the consortium for their commitment to data sharing. Interpretations are the responsibility of study authors.

\section{Statement of Ethics}

The authors have no ethical conflicts to disclose.

\section{Disclosure Statement}

The authors declare no conflict of interest.

\section{Author Contributions}

All the authors equally contributed to the preparation of this manuscript.

\section{References}

1 Kleeff J, Korc M, Apte M, La Vecchia C, Johnson CD, Biankin AV, et al. Pancreatic cancer. Nat Rev Dis Primers. 2016 Apr;2:16022.

2 Integrated Genomic Characterization of Pancreatic Ductal Adenocarcinoma. Cancer Cell (2017) 32:185-203 e13. 
3 NCCN Clinical Practice Guidelines In Oncology - Pancreatic Adenocarcinoma. (version 3.2017). (Accessed at February 1, 2018) https://www.nccn.org/professionals/physician_gls/pdf/pancreatic.pdf)

4 Turski ML, Vidwans SJ, Janku F, Garrido-Laguna I, Munoz J, Schwab R, et al. Genomically Driven Tumors and Actionability across Histologies: BRAF-Mutant Cancers as a Paradigm. Mol Cancer Ther. 2016 Apr;15(4):533-47.

5 Hainsworth JD, Meric-Bernstam F, Swanton C, Hurwitz H, Spigel DR, Sweeney C, et al. Targeted Therapy for Advanced Solid Tumors on the Basis of Molecular Profiles: Results From MyPathway, an Open-Label, Phase IIa Multiple Basket Study. J Clin Oncol. 2018 Feb;36(6):536-42.

6 Hyman DM, Puzanov I, Subbiah V, Faris JE, Chau I, Blay JY, et al. Vemurafenib in Multiple Nonmelanoma Cancers with BRAF V600 Mutations. N Engl J Med. 2015 Aug;373(8):726-36.

7 Mulligan LM. RET revisited: expanding the oncogenic portfolio. Nat Rev Cancer. 2014 Mar;14(3):173-86.

8 Middleton G, Palmer DH, Greenhalf W, Ghaneh P, Jackson R, Cox T, et al. Vandetanib plus gemcitabine versus placebo plus gemcitabine in locally advanced or metastatic pancreatic carcinoma (ViP): a prospective, randomised, double-blind, multicentre phase 2 trial. Lancet Oncol. 2017 Apr;18(4):486-99.

9 Landrum MJ, Lee JM, Benson M, Brown GR, Chao C, Chitipiralla S, et al. ClinVar: improving access to variant interpretations and supporting evidence. Nucleic Acids Res. 2018 Jan;46 D1:D1062-7.

10 Corcoran RB, Atreya CE, Falchook GS, Kwak EL, Ryan DP, Bendell JC, et al. Combined BRAF and MEK Inhibition With Dabrafenib and Trametinib in BRAF V600-Mutant Colorectal Cancer. J Clin Oncol. 2015 Dec;33(34):4023-31.

11 Planchard D, Smit EF, Groen HJ, Mazieres J, Besse B, Helland Å, et al. Dabrafenib plus trametinib in patients with previously untreated BRAFV600E-mutant metastatic non-small-cell lung cancer: an open-label, phase 2 trial. Lancet Oncol. 2017 Oct;18(10):1307-16.

12 Robert C, Karaszewska B, Schachter J, Rutkowski P, Mackiewicz A, Stroiakovski D, et al. Improved overall survival in melanoma with combined dabrafenib and trametinib. N Engl J Med. 2015 Jan;372(1):30-9.

13 Drilon A, Hu ZI, Lai GG, Tan DS. Targeting RET-driven cancers: lessons from evolving preclinical and clinical landscapes. Nat Rev Clin Oncol. 2018 Mar;15(3):151-67.

14 Grinshpun A, Gavert N, Granit RZ, Masuri H, Ben-Porath I, Breuer S, et al. Ev vivo organ culture as potential prioritization tool for breast cancer targeted therapy. Cancer Biol Ther. 2018 Aug;19(8):645-8.

15 AACR Project GENIE Consortium. AACR Project GENIE: Powering Precision Medicine through an International Consortium. Cancer Discov. 2017 Aug;7(8):818-31.

16 Geller LT, Barzily-Rokni M, Danino T, Jonas OH, Shental N, Nejman D, et al. Potential role of intratumor bacteria in mediating tumor resistance to the chemotherapeutic drug gemcitabine. Science. 2017 Sep;357(6356):1156-60.

17 Bailey P, Chang DK, Forget MA, Lucas FA, Alvarez HA, Haymaker C, et al. Exploiting the neoantigen landscape for immunotherapy of pancreatic ductal adenocarcinoma. Sci Rep. 2016 Oct;6(1):35848.

18 Gold KA. ROS1 -targeting the one percent in lung cancer. N Engl J Med. 2014 Nov;371(21):2030-1. 


\section{Case Reports in Oncology}

Case Rep Oncol 2019;12:7-13
\begin{tabular}{l|l}
\hline DOI: 10.1159/000496018 & $\begin{array}{l}\text { (c) 2019 The Author(s). Published by S. Karger AG, Basel } \\
\text { www.karger.com/cro }\end{array}$ \\
\hline
\end{tabular}

Grinshpun et al.: Beyond KRAS: Practical Molecular Targets in Pancreatic Adenocarcinoma

Table 1. BRAF and RET mutations as described in two large public databases\#

\begin{tabular}{|c|c|c|c|c|}
\hline Gene & Database & Protein change & Mutation class & Pathogenic @ ClinVar \\
\hline \multirow[t]{13}{*}{ BRAF } & \multirow[t]{8}{*}{ TCGA } & TV207TV & Insertion & - \\
\hline & & T488_Q493delinsK & In Frame deletion & - \\
\hline & & V600E & Missense & + \\
\hline & & V600E & Missense & + \\
\hline & & V600E & Missense & + \\
\hline & & V471I & Missense & + \\
\hline & & V487S & Missense & - \\
\hline & & $\mathrm{R} 462 \mathrm{~T}$ & Missense & - \\
\hline & \multirow[t]{5}{*}{ GENIE } & Y633H & Missense & - \\
\hline & & $* 767$ Cext*3 & Nonstop mutation & - \\
\hline & & A598A & Silent mutation & - \\
\hline & & V600_K601delinsE & In Frame deletion & - \\
\hline & & N486_P490del & In Frame deletion & - \\
\hline \multirow[t]{14}{*}{ RET } & \multirow[t]{12}{*}{ TCGA } & R844W & Missense & - \\
\hline & & A756D & Missense & - \\
\hline & & $\mathrm{R} 770 *$ & Nonsense & - \\
\hline & & R57W & Missense & - \\
\hline & & A1019V & Missense & - \\
\hline & & A1019V & Missense & - \\
\hline & & A1019V & Missense & - \\
\hline & & G1086A & Missense & - \\
\hline & & PS766PS & In Frame & - \\
\hline & & F329L & Missense & - \\
\hline & & P1070S & Missense & - \\
\hline & & R57Q & Missense & - \\
\hline & \multirow[t]{2}{*}{ GENIE } & P992L & Missense & - \\
\hline & & $\mathrm{H} 784 \mathrm{H}$ & Silent mutation & - \\
\hline
\end{tabular}

\# Accessed March 2018. 